\title{
Restrained 2-Domination in Graphs
}

\author{
Indrani Pramod Kelkar ${ }^{1}$, J Jagan Mohan ${ }^{2}$ \\ ${ }^{1}$ Professor, Department of Mathematics, Acharya Institute of Technology, Bangalore \\ ${ }^{2}$ Assistant Professor, VIT University, Vellore
}

\begin{abstract}
Let $G=(V, E)$ be a graph. A set $S \subseteq V(G)$ is a restrained 2- dominating set if every vertex of $V(G) \backslash S$ is adjacent to at least two vertices in $S$ and every vertex of $V(G) \backslash S$ is adjacent to a vertex in $V(G) \backslash S$. The restrained 2- domination number of $G$, denoted by $\gamma_{r 2}(G)$, is the smallest cardinality of a restrained 2- dominating set of $G$. In this paper we study restrained 2-domination in graphs and obtain some results.
\end{abstract}

Keywords: - Domination, 2-domination, restrained domination 2000 Mathematics Subject Classification: 05 C69

\section{Introduction}

Let $\mathrm{G}=(\mathrm{V}, \mathrm{E})$ be a finite undirected graph without loops or multiple edges. The order and size of $\mathrm{G}$ are denoted by $p$ and $q$ respectively. For graph theoretical terms we refer to Harary [4] and for terms related to domination we refer to Haynes et al. [9]. For every vertex $v \in V$, the open neighborhood $\mathrm{N}(v)$ is the set $\{u \in \mathrm{V}(\mathrm{G}) / u v \in \mathrm{E}(\mathrm{G})\}$ and the closed neighborhood $\mathrm{N}[\mathrm{v}]$ is the set $\mathrm{N}[\mathrm{v}]=\mathrm{N}(\mathrm{v}) \mathrm{U}\{\mathrm{v}\}$. A vertex in a graph $\mathrm{G}$ dominates itself and its neighbors. An end-vertex or a pendant vertex in a graph $\mathrm{G}$ is a vertex of degree one and a support vertex is one that is adjacent to an end-vertex.

A subset $\mathrm{S}$ of $\mathrm{V}$ in a graph $\mathrm{G}$ is said to be a dominating set of $\mathrm{G}$ if every vertex of $V(G) \backslash S$ is adjacent to at least one vertex in $\mathrm{S}$. The domination number $\gamma(\mathrm{G})$ is the minimum cardinality of a dominating set of G. A dominating set $\mathrm{S}$ with $|S|=\gamma$ is called a $\gamma$-set.

The concept of restrained domination was introduced by Telle and Proskurowski [5] indirectly as a vertex partitioning problem. Cyman and Raczek [6] introduced the concept of total restrained domination.

A dominating set $\mathrm{S} \subseteq \mathrm{V}(\mathrm{G})$ is a restrained dominating set if every vertex of $V(G) \backslash S$ is adjacent to a vertex in $S$ and every vertex of $V(G) \backslash S$ is adjacent to a vertex in $V(G) \backslash S$. The restrained domination number of $\mathrm{G}$ denoted by $\gamma_{r}(\mathrm{G})$ is the minimum cardinality of a restrained dominating set of $\mathrm{G}$. A restrained dominating set $\mathrm{S}$ with $|S|=\gamma_{r}$ is called a $\gamma_{r}$-set.

The concept of 2-domination was introduced by Fink and Jacobson [1, 2].

A dominating set $\mathrm{S} \subseteq \mathrm{V}(\mathrm{G})$ is a 2-dominating set if every vertex of $V(G) \backslash S$ is adjacent to at least two vertices in $\mathrm{S}$. The 2-domination number of $\mathrm{G}$ denoted by $\gamma_{2}(\mathrm{G})$ is the minimum cardinality of a 2-dominating set of G. A restrained dominating set $\mathrm{S}$ with $|S|=\gamma_{2}$ is called a $\gamma_{2}$-set.

In this paper we study restrained 2-domination in graphs and obtain some results.

\section{Main Results}

A set $\mathrm{S} \subseteq \mathrm{V}(\mathrm{G})$ is a restrained 2-dominating set of $\mathrm{G}$ if every vertex of $V(G) \backslash S$ is adjacent to at least two vertices in $\mathrm{S}$ and to a vertex in $V(G) \backslash S$. The restrained 2-domination number of $\mathrm{G}$ denoted by $\gamma_{r 2}(\mathrm{G})$ is the minimum cardinality of a restrained 2-dominating set of $G$. A restrained 2- dominating set of cardinality $\gamma_{r 2}(G)$ is called a $\gamma_{r 2}(G)$-set.

Let $\mathrm{S}$ be any restrained 2-dominating set of a graph G. Then the degree of every vertex of $V(G) \backslash S$ is at least three and all the vertices of degree less than three must be included in $\mathrm{S}$. Thus $\mathrm{S}$ contains all pendant vertices and its supporting vertices.

If $\mathrm{p}$ is the number of vertices in $\mathrm{G}$ then bounds for $\gamma_{r 2}(G)$ can be stated as: 
Lemma 2.1: For any graph $\mathrm{G}, 2 \leq \gamma_{r 2}(G) \leq p$.

Lemma 2.2: For any graph $\mathrm{G}$ with $\delta(G) \geq 3, \gamma_{r 2}(G) \leq p-2$.

Since any restrained 2-dominating set is also a 2-dominating set, so the inequality $\gamma_{2}(G) \leq \gamma_{r 2}(G)$ for any graph $G$ is obvious. On the other hand if a graph $\mathrm{G}$ has a $\gamma_{2}(G)$ set S such that $\mathrm{G}[V(G) \backslash S]$ has no isolated vertices, then $\mathrm{S}$ is also a restrained 2- dominating set, so $\gamma_{r 2}(G) \leq \gamma_{2}(G)$.

Hence we have the following characterization of those graphs for which the 2- restrained domination number is equal to the 2 -domination number.

Theorem 2.3: For a graph G, $\gamma_{2}(G)=\gamma_{r 2}(G)$ if and only if $\mathrm{G}$ has a $\gamma_{2}(G)$-set S such that $\mathrm{G}[V(G) \backslash S]$ has no-isolated vertices.

Suppose that $n \geq 1$ and let $k \in\{1,2, \ldots n-2, n-1, n\}$. Let $\mathrm{G}$ be the graph obtained from $P_{n-k}$, the path on $n-k$ vertices, by adding a set of vertices $\left\{u, v, v_{1}, v_{2}, \ldots v_{k-1}\right\}$ and joining the vertices $u$ and $v$ to each of the vertices in $V\left(P_{n-k}\right) \cup\left\{v_{1}, v_{2}, \ldots v_{k-1}\right\}$. Then $\gamma_{2}(G)=2$ and $\gamma_{r 2}(G)=k$. Hence we have the result-

Theorem 2.4: There exists a graph $\mathrm{G}$ for which $\gamma_{r 2}(G)-\gamma_{2}(G)$ can be made arbitrarily large.

Let $K_{n}, C_{n}$ and $P_{n}$ denote, respectively, the complete graph, the cycle graph and the path graph of order n. Also, let $W_{n}$ and $F_{n}$ denote the wheel graph and fan graph of order n. $K_{m, n}$ is a complete bipartite graph on $m$ and $n$ vertices and $K_{1, n-1}$ a star graph of order n. A galaxy is a disjoint union of stars. A bistar $\mathrm{B}(\mathrm{r}, \mathrm{s})$ is a graph obtained by joining the centres of two stars $K_{1, r}$ and $K_{1, s}$ by an edge.

If a vertex is of degree 2 in $G$ then both of its adjacent vertices must be included in restricted 2dominating set $\mathrm{S}$.

\section{Theorem 2.5:}

(i) If $n$ is a positive integer, then $\gamma_{r 2}\left(K_{n}\right)=n$ for $\mathrm{n} \leq 3$

(ii) If $n \geq 3$ is a positive integer, then $\gamma_{r 2}\left(C_{n}\right)=n$.

(iii) If $n$ is a positive integer, then $\gamma_{r 2}\left(P_{n}\right)=n$.

(iv) If $n \geq 4$ is a positive integer, then $\gamma_{r 2}\left(W_{n}\right)=\left\lfloor\frac{n}{2}\right\rfloor$.

(v) If $n \geq 4$ is a positive integer, then $\gamma_{r 2}\left(F_{n}\right)=\left\lceil\frac{\mathrm{n}}{2}\right\rceil$.

(vi) For any positive integers $m$ and $n, \gamma_{r 2}\left(K_{m, n}\right)=4 \quad$ for $\mathrm{m} \geq 3$ and $\mathrm{n} \geq 3$

$$
=m+n \text { otherwise. }
$$

(vii) If $n \geq 2$ is a positive integer, then $\gamma_{r 2}\left(K_{1, n-1}\right)=n$.

(viii) For a Bistar,

$$
\begin{aligned}
\gamma_{r 2}[B(r, s)] & =r+s & & \text { for } \mathrm{r} \geq 2 \text { and } \mathrm{s} \geq 2 \\
& =r+s+2 & & \text { otherwise. }
\end{aligned}
$$

Proof : (i) Domination number of complete graph is one and 2-domination number is 2 . A vertex of $K_{n}$ is adjacent to all the remaining vertices, so for $\mathrm{n}>3$ a minimal 2 - dominating set is a minimal restrained 2-dominating set, hence the result.

ii, iii) For $\mathrm{C}_{\mathrm{n}}$ and $\mathrm{P}_{\mathrm{n}}$ as max degree of a vertex is 2, every vertex must be included in the restrained 2-dominating set. Hence the result.

iv) For $\mathrm{W}_{\mathrm{n}}, \mathrm{n}>3$, the center vertex must be in the 2-dominating set. From the cycle $\mathrm{C}_{\mathrm{n}-1}$, we take alternate set of the vertices in restrained 2-dominating set. Hence the result. 
vi) For $\mathrm{Km}, \mathrm{n}$ two vertices each from two parts is sufficient fot restrained 2-domination for $\mathrm{m}, \mathrm{n}>=3$, otherwise we must include all the vertices. Similarly for star graph we must include all vertices into restrained 2dominating set as except the central vertex, all vertices are of degree $<2$. Also for bi-star similar argument follows.

Now we determine the restrained 2-domination number of the complements of some families of graphs mentioned above. Here $\bar{G}$ denotes the complement of a graph G.

\section{Theorem 2.6:}

(i) If $n$ is a positive integer, then for complement of complete Graphs $\mathrm{K}_{\mathrm{n}} \quad \gamma_{r 2}\left(\bar{K}_{n}\right)=n$.

(ii) If $n \geq 3$ is a positive integer, then for complement of cycle graph $\mathrm{C}_{\mathrm{n}} \begin{array}{r}\gamma_{r 2}\left(\bar{C}_{n}\right)=n \text { for } \mathrm{n} \leq 5 \\ =3 \text { for } \mathrm{n}>5 .\end{array}$

(iii) If $n$ is a positive integer, then for complement of path graph $\mathrm{P}_{\mathrm{n}} \quad \begin{aligned} \gamma_{r 2}\left(\bar{P}_{n}\right)=n & \text { for } \mathrm{n} \leq 4 \\ & =4 \text { for } \mathrm{n}>4\end{aligned}$

$$
=4 \text { for } \mathrm{n}>4 \text {. }
$$

(iv) If $n \geq 3$ is a positive integer, then for complement of wheel graph $\mathrm{W}_{\mathrm{n}} \gamma_{r 2}\left(\bar{W}_{n}\right)=n$ for $\mathrm{n} \leq 6$

$$
=4 \text { for } \mathrm{n}>6 \text {. }
$$

(v) If $n \geq 3$ is a positive integer, then for complement of fan graph $\mathrm{F}_{\mathrm{n}} \quad \gamma_{r 2}\left(\bar{F}_{n}\right)=n$ for $\mathrm{n} \leq 5$

$$
=4 \text { for } \mathrm{n}>5 \text {. }
$$

(vi) If $\mathrm{m}, \mathrm{n}$ are positive integers, then for complement of complete bipartite graph

$$
\begin{aligned}
\gamma_{r 2}\left(\bar{K}_{m, n}\right) & =m+n & & \text { for } \mathrm{m} \leq 3 \text { and } \mathrm{n} \leq 3 \\
& =4 & & \text { for } \mathrm{m} \geq 4 \text { and } \mathrm{n} \geq 4 \\
& =5 & & \text { otherwise. }
\end{aligned}
$$

(vii) If $n$ is a positive integer, then for complement of star graph $\mathrm{K}_{1, \mathrm{n}} \gamma_{r 2}\left(\bar{K}_{1, n-1}\right)=4$ for $\mathrm{n}=4$

(viii) For the complement of Petersen graph G, $\gamma_{r 2}(\bar{G})=4$.

$$
=3 \text { otherwise. }
$$

(ix) For complement of Bistar B(r,s), $\quad \gamma_{r 2}[\bar{B}(r, s)]=r+s+1$ for $(\mathrm{r}, \mathrm{s}) \quad(0,0),(1$ From the above results we can get the following equalities-

$$
=3 \quad \text { otherwise. }
$$

\section{Remark 2.7:}

(i) If $n \geq 4$ is a positive integer, then $\gamma_{r 2}\left(\bar{W}_{n}\right)=1+\gamma_{r 2}\left(\bar{C}_{n-1}\right)$.

(ii) If $n \geq 4$ is a positive integer, then $\gamma_{r 2}\left(\bar{F}_{n}\right)=1+\gamma_{r 2}\left(\bar{P}_{n-1}\right)$.

(iii) For any positive integers $m$ and $n, \gamma_{r 2}\left(\bar{K}_{m, n}\right)=\gamma_{r 2}\left(K_{m}\right)+\gamma_{r 2}\left(K_{n}\right)$.

(iv) If $n \geq 2$ is a positive integer, then $\gamma_{r 2}\left(\bar{K}_{1, n-1}\right)=1+\gamma_{r 2}\left(K_{n-1}\right)$.

We can prove the inequality for the cross product of two graphs $\mathrm{G}$ and $\mathrm{H}$ if the two graphs posses $\gamma_{r 2}(G)$-set and a $\gamma_{r 2}(H)$-set.

Theorem 2.8: $\quad$ For any two graphs $\mathrm{G}$ and $\mathrm{H}, \gamma_{r 2}(G \times H) \leq \gamma_{r 2}(G) \gamma_{r 2}(H)$ where $G \times H$ denote the cross product of $\mathrm{G}$ and $\mathrm{H}$.

Proof: Let D be a $\gamma_{r 2}(G)$-set and $D^{\prime}$ be a $\gamma_{r 2}(H)$-set.

Let $(u, v) \in G \times H$. Then there exist at least two vertices, respectively, $a, b \in D$ and $a^{\prime}, b^{\prime} \in D^{\prime}$ such that $a, b$ are adjacent to $u$ and $a^{\prime}, b^{\prime}$ are adjacent to $v$. Thus $\left(a, a^{\prime}\right),\left(b, b^{\prime}\right) \in D \times D^{\prime}$ dominates $(u, v) \in G \times H$. Now let $x \in G \backslash D$ and $y \in H \backslash D^{\prime}$. Then there 
exist at least one vertex, respectively, $c \in G \backslash D$ and $d \in H \backslash D^{\prime}$ such that $c$ is adjacent to $x$ and $d$ is adjacent to $y$. Thus $(x, y) \in(G \times H) \backslash\left(D \times D^{\prime}\right)$ is adjacent to $(c, d) \in(G \times H) \backslash\left(D \times D^{\prime}\right)$. Hence $D \times D^{\prime}$ is a restrained 2-dominating set for $G \times H$. Since $\left|D \times D^{\prime}\right| \leq|D|\left|D^{\prime}\right|$, the theorem follows.

Theorem 2.9: If $\mathrm{T}$ is a tree, then $\gamma_{r 2}(T) \geq 1+\Delta(T)$. Moreover the equality holds if and only if $\mathrm{T}$ is a star.

\section{References}

[1] Fink J.F., Jacobson M.S., n-Domination in Graphs, in: Alavi Y. and Schwenk A. J.(eds), Graph Theory with Applications to Algorithms and Computer Science, New York, Wiley, 1985, 283-300.

[2] Fink J.F., Jacobson M.S., On n-Domination, n-Dependence and Forbidden Subgraphs, Graph Theory with Applications to Algorithms and Computer Science, New York, Wiley, 1985, 301-312.

[3] G.S.Domke, J.H.Hattingh, S.T.Hedetniemi, R.C.Laskar and L.R.Markus, Restrained Domination in Graphs, Discrete Math., 203 (1999), 61-69.

[4] Harary F., Graph Theory, Addison-Wesley, Reading, Mass, 1972.

[5] J.A.Telle and A.Proskurowski, Algorithms for Vertex Partitioning Problems on Partial-k Trees, SIAM J. Discrete Mathematics, 10 (1997), 529-550.

[6] J.Cyman and J.Raczek, On the Total Restrained Domination Number of a Graph, Australas J. Combin., 36 (2006), 91-100.

[7] Nader Jafari Rad, Results on Total Restrained Domination in Graphs, Int. J. Contemp. Math. Sciences, Vol.3, 2008, No.8, $383-387$.

[8] T.W.Haynes, S.T.Hedetniemi and P.J.Slater, Editors, Fundamental of Domination in Graphs: Advanced Topics, (Marcel Dekker, Inc, New York, (NY), 1998 